\title{
How to Take the Removal of Condensation Water into Account in Polyesterification Kinetics?
}

\author{
Qiquan Chen, ${ }^{\dagger}$ Hui Chang, and Dong $\mathrm{ZHANG}^{\dagger \dagger}$ \\ Department of Petrochemical Engineering, Fushun Petroleum Institute, \\ Fushun 113001, People's Republic of China
}

(Received January 26, 1999)

\begin{abstract}
In previous polyesterification reactions, the concentrations of reactants and catalyst (if present) were all expressed in moles per kilogram. In most cases, the progress of reaction was followed by the titration of acid groups. Consequently, the removal of condensation water is bound to affect the concentrations of reactants and catalyst. In this paper, a new general kinetic equation has been derived, which takes this effect due to the removal of condensation water into consideration more accurately. The unit of moll-1, who replaced the unit of $\mathrm{mol} \mathrm{kg}^{-1}$, is led into the calculation of acid concentration and the derivation of the general polyesterification kinetic equation for the first time. Its adoption can finally eliminate the error due to the change of the density of reaction system while adopting the unit of mol kg-1. KEY WORDS Polyesterification/Kinetic Equation/Removal of Condensation Water /
\end{abstract}

In most polyesterification reactions, the progress of reaction is followed by the titration of acid groups at a given time. But the volume and the weight of reaction system are varied with the removal of condensation water successively. As a result, their concentrations can be affected by the removal of condensation water. In consequence, many authors tried to find out how to eliminate this effect in kinetic data treatment.

In 1971, Szabo-Rethy firstly showed that the rate constant calculated by Flory's method could result in an error of $15 \%$ to $35 \%$, which neglected the effect of the removal of condensation water. ${ }^{1,2}$ And he gave a calculating method that takes the removal of condensation water into account. After that, Lin and Hsieh also obtained similar relation. ${ }^{3}$ In 1980, Fradet and Maréchal derived a general kinetic equation that takes water removal into consideration. ${ }^{4}$ They concluded that if the concentrations instead of the extents of reaction are used in kinetic equations, no correction is needed for stoichiometric reaction; when the balance of reactants is non-stoichiometric, the concentrations of reactants must be corrected. On the basis of them, Hu and Wang developed a more complete kinetic equation that takes water removal into account while adopting the unit of $\mathrm{mol} \mathrm{kg}^{-1}$. $^{5}$ They considered that the removal of condensation water should be taken into account not only for the concentrations of reactants, but also for that of catalyst if it contributes to the overall rate of reaction. And they also pointed out that the rate constant obtained using $\mathrm{mol} \mathrm{kg}^{-1}$, as the unit of concentration is not a real constant but a function of the density of reaction mixture. However, during the course of derivation of a general kinetic equation, they made a rough assumption. Namely, the condensation water was assumed to be completely and instaneously removed from the reaction system. In fact, complete removal is very difficult. In some investigation, the amount of water remained in reaction system can be as high as $41 \%$ of

\footnotetext{
† Present Address: Jiujiang Petrochemical General Works, SINOPEC, Jiujiang 332004, People's Republic of China.

${ }^{\dagger}$ To whom correspondence should be addressed.
}

the total water produced. ${ }^{6}$ Obviously, their assumption is not appropriate. Therefore, the treating result by their equation may lead to appreciable error.

The aim of this paper is to develop a general kinetic equation of polyesterification reaction while adopting the unit of $\mathrm{moll}^{-1}$. Its derivation will completely eliminate the error in kinetic data treatment, which comes from the removal of condensation water during the course of polyesterification reaction.

\section{THEORETICAL}

\section{Comparison of the Concentrations Expressed in $\mathrm{Mol} \mathrm{kg}^{-1}$} and $\mathrm{Moll}^{-1}$ in Kinetic Equation

Expressing concentration in $\mathrm{mol} \mathrm{kg}^{-1}$ and $\mathrm{moll}^{-1}$ are only two different forms of expression. But this difference may lead to appreciable error in the analysis of kinetic data of polyesterification reactions, as is demonstrated below.

Starting with the following general kinetic equation,

$$
\frac{\mathrm{d}[\mathrm{COOH}]}{\mathrm{d} t}=K[\mathrm{Cat}]^{z}[\mathrm{COOH}]^{x}[\mathrm{OH}]^{y}
$$

Here $x, y$, and $z$ are the reaction orders relative to the concentrations of acid, alcohol and catalyst, respectively; $K$ is the rate constant. When no external catalysts are present or when overall reaction rate is independent of catalyst concentration, $z=0$.

We can turn eq 1 into other two forms of eq 2 or eq 3 :

$$
\begin{aligned}
& -\frac{1}{V} \frac{\mathrm{d} N_{\mathrm{a}}}{\mathrm{d} t}=K_{\mathrm{V}}\left(\frac{N_{\mathrm{c}}}{V}\right)^{z}\left(\frac{N_{\mathrm{a}}}{V}\right)^{x}\left(\frac{N_{\mathrm{b}}}{V}\right)^{y} \\
& -\frac{1}{M} \frac{\mathrm{d} N_{\mathrm{a}}}{\mathrm{d} t}=K_{\mathrm{M}}\left(\frac{N_{\mathrm{c}}}{M}\right)^{z}\left(\frac{N_{\mathrm{a}}}{M}\right)^{x}\left(\frac{N_{\mathrm{b}}}{M}\right)^{y}
\end{aligned}
$$

Here $N_{\mathrm{a}}, N_{\mathrm{b}}$, and $N_{\mathrm{c}}$ are the molar quantities of acid, alcohol, and catalyst at time $t$, respectively; $V$ and $M$ denote the total volume and mass of the reaction mixture at time $t$, and the coefficients $K_{\mathrm{V}}$ and $K_{\mathrm{M}}$ represent volume and mass reaction rate constants, respectively. 
Dividing eq 2 by eq 3 , we can obtain eq 4 :

$$
K_{\mathrm{M}}=K_{\mathrm{V}}\left(\frac{M}{V}\right)^{x+y+z-1}=K_{\mathrm{V}} d^{x+y+z-1}
$$

Here $d$ is the density of the reaction mixture at time $t$.

From this equation, we can find out that $K_{\mathrm{M}}$ is not a real constant but a function of the density while adopting mass concentrations in kinetic equation. The density of a polyesterification reaction system usually increases with the extent of reaction because of the removal of the condensation water. Therefore the rate constant $K_{\mathrm{M}}$ should not be a constant but an increasing function of the degree of reaction. As a result, it is necessary to consider the removal of water and change in density simultaneously. In order to obtain true rate constant, it is essential to use volume concentrations instead of mass concentrations.

Calculation of Acid Concentration and Derivation of a General Kinetic Equation while Adopting the Unit of $\mathrm{Moll}^{-1}$

In most polyesterification reactions, the titration method of acid groups is used to follow the progress of the reaction. In order to develop a general kinetic equation that considers water removal while adopting the unit of $\mathrm{moll}^{-1}$, the unit of acid concentration should firstly be changed to $\mathrm{moll}^{-1}$ from $\mathrm{mol} \mathrm{kg}^{-1}$.

As we know, the calculating equation of acid value $(A V)$ usually can be expressed as follows:

$$
A V=\frac{56.1 \times V_{\mathrm{KOH}} \times C_{\mathrm{KOH}}}{W_{\text {sample }}}\left(\mathrm{mg} \mathrm{KOH} \mathrm{g}^{-1}\right)
$$

Here $V_{\text {кон }}$ stands for the volume of the titration with $\mathrm{KOH}$ solution $(\mathrm{ml}) ; C_{\mathrm{KOH}}$ denotes the concentration of $\mathrm{KOH}$ solution $\left(\mathrm{moll}^{-1}\right) ; W_{\text {sample }}$ is the weight of sample taken from the reaction system at time $t$.

As far as diacid is concerned, its concentration at time $t$ can be calculated by the following equation:

$$
C_{t}=\frac{A V}{56.1} \times \frac{1}{2}\left(\mathrm{~mol} \mathrm{~kg}^{-1}\right)
$$

In this, the unit of acid concentration $\left(C_{t}\right)$ is $\mathrm{mol} \mathrm{kg}^{-1}$. In order to obtain its volume concentration at time $t$, we should firstly get the density of reaction system at time $t$ according to the following equation:

$$
d_{t}=\frac{W_{t}}{V_{t}}=\frac{W_{0}-W_{t, \mathrm{sample}}-W_{t, \mathrm{H}_{2} \mathrm{O}}}{V_{0}-V_{t, \mathrm{sample}}-V_{t, \mathrm{H}_{2} \mathrm{O}}}\left(\mathrm{kg}^{-1}\right)
$$

Here $W_{t}, V_{t}$ are the mass and volume of reaction mixture at time $t$, respectively; $W_{t \text {,sample }}$ denotes the sample's total mass taken from the reaction system from the initial time to the time $t, V_{t \text {,sample }}$ represents its volume, which can be estimated by using a graduated sampling tube; $V_{t, \mathrm{H}_{2} \mathrm{O}}$ is the total volume of the condensation water that is removed from the reaction system into the oilwater separator by the time $t$, it can be read from the graduation of the oil-water separator, $W_{t, \mathrm{H}_{2} \mathrm{O}}$ stands for its weight. If the density of water is considered as $1 \mathrm{~g} \mathrm{~mL}^{-1}, W_{t, \mathrm{H}_{2} \mathrm{O}}$ in quantity is equal to $V_{t, \mathrm{H}_{2} \mathrm{O}} ; W_{0}$ is the total mass of reactants before mixing, $V_{0}$ denotes its volume. If neglecting the effect of the partial molar quantity, it can be calculated as follows:

$$
V_{0}=\frac{W_{\mathrm{a}}}{d_{\mathrm{a}}}+\frac{W_{\mathrm{b}}}{d_{\mathrm{b}}}+\cdots
$$

Here $W$ and $d$ stand for the weight and density of every reactant at reaction temperature, respectively.

Multiplying eq 6 by eq 7, yields:

$$
C_{t}^{\prime}=C_{t} \cdot d_{t}=\frac{A V}{56.1} \times \frac{1}{2} \times \frac{W_{0}-W_{t, \text { sample }}-W_{t, \mathrm{H}_{2} \mathrm{O}}}{V_{0}-V_{t, \mathrm{sample}}-V_{t, \mathrm{H}_{2} \mathrm{O}}}
$$

Up to this, the acid concentration has been changed to volume concentration $\left(C_{t}^{\prime}\right)$ from mass concentration $\left(C_{t}\right)$.

Suppose the initial concentrations $\left(\mathrm{moll}^{-1}\right)$ of acid, alcohol, and catalyst are $a_{0}, b_{0}$, and $c_{0}$, respectively; and their concentrations at time $t$ are $a, b$, and $c$. In general, only acid is titrated and the alcohol concentration is obtained by the calculation. When condensation water is removed from the reaction system, the number of hydroxyl groups in excess does not change although the concentration of excess of the hydroxyl groups increases with the removal of condensation water. Thus:

$$
\begin{gathered}
\left(b_{0}-a_{0}\right) V_{0}=(b-a) V_{t} \\
b=a+\frac{V_{0}}{V_{t}}\left(b_{0}-a_{0}\right)
\end{gathered}
$$

Likewise, the mole number of catalyst should keep constant,

$$
C_{0} V_{0}=C V_{t}
$$

Then, the catalyst concentration at time $t$ is:

$$
C=\frac{V_{0}}{V_{t}} C_{0}
$$

Therefore, the general kinetic equation taking the water removal into consideration is:

$$
-\frac{\mathrm{d} a}{\mathrm{~d} t}=K^{\prime}\left(\frac{V_{0}}{V_{t}} C_{0}\right)^{z} a^{x}\left(a+\frac{V_{0}}{V_{t}}\left(b_{0}-a_{0}\right)\right)^{y}
$$

$K^{\prime}$ is the corrected reaction rate constant.

The general kinetic equation without taking water removal into account can be expressed:

$$
-\frac{\mathrm{d} a}{\mathrm{~d} t}=K C_{0}^{z} a^{x}\left(a+b_{0}-a_{0}\right)^{y}
$$

$K$ is the uncorrected reaction rate constant.

Dividing eq 14 by eq 15 , we thus obtain:

$$
\frac{K^{\prime}}{K}=\left(\frac{V_{t}}{V_{0}}\right)^{z}\left(\frac{a+b_{0}-a_{0}}{a+\frac{V_{0}}{V_{t}}\left(b_{0}-a_{0}\right)}\right)^{y}
$$

If $b_{0}>a_{0}$, it is not difficult to find out that the ratio $K^{\prime} / K$ is always less then unity. This conclusion is the same as Hu's report. ${ }^{5}$ Of course, if the reactants and catalyst can be titrated independently during the course of the polyesterification reaction, the correction is unnecessary. 


\section{CONCLUSION}

A new general kinetic equation has been derived while adopting the unit of $\mathrm{moll}^{-1}$. First, it takes the change of the concentration of reactants and catalyst (if present) due to the removal of the condensation water into account more accurately. Secondly, it eliminated the effect of the density change of reaction mixture because the unit of moll-1 instead of the unit of $\mathrm{mol} \mathrm{kg}^{-1}$ is used. Therefore, the rate constant obtained by the new general kinetic equation is true rate constant. For the case of the discussion of the kinetic behavior of polyesterification reaction, its employment is of more practical significance.

\section{REFERENCES}

1. E. Szabo-Rethy, Eur. Polym. J., 7, 1485 (1971).

2. P. J. Flory, J. Am. Chem. Soc., 61, 3334 (1939).

3. C. C. Lin and R. H. Hsieh, J. Appl. Polym. Sci., 21, 2711 (1977).

4. A. Fradet and E. Maréchal, Polym. Bull., 3, 441 (1980).

5. G. H. Hu and W. Wang, Polym. Bull., 28, 351 (1992).

6. S. A. Chen and K. C. Wu, J. Polym. Sci., Polym. Chem. Ed., 20, 1819 (1982) 